\title{
THE BOUNDARY BEHAVIOR OF HOLOMORPHIC FUNCTIONS: GLOBAL AND LOCAL RESULTS*
}

\author{
STEVEN G. KRANTZ ${ }^{\dagger}$
}

This paper is dedicated to Salah Baouendi, with much respect and admiration

\begin{abstract}
We develop a new technique for studying the boundary limiting behavior of a holomorphic function on a domain $\Omega$ - both in one and several complex variables. The approach involves two new localized maximal functions.

As a result of this methodology, theorems of Calderón type about local boundary behavior on a set of positive measure may be proved in a new and more natural way.

We also study the question of nontangential boundedness (on a set of positive measure) versus admissible boundedness. Under suitable hypotheses, these two conditions are shown to be equivalent.
\end{abstract}

Key words. Fatou theorem, admissible convergence, Caldéron theorem, and boundary limits

AMS subject classifications. Primary 32A40; Secondary 32A35, 32A50

0. Introduction. The first theorem about the boundary limiting behavior of holomorphic functions was proved by P. Fatou in his thesis in 1906 [FAT]. He used Fourier series techniques to show that if $f$ is a bounded, holomorphic function on the unit disc $D \subseteq \mathbb{C}$ (i.e., $f \in H^{\infty}(D)$ ), then

$$
\lim _{r \rightarrow 1^{-}} f\left(r e^{i \theta}\right)
$$

exists for almost every $\theta \in[0,2 \pi)$. Furthermore, for $\alpha>1$ and $P \in \partial D$, we define

$$
\Gamma_{\alpha}(P)=\{\zeta \in D:|z-P|<\alpha(1-|z|)\} .
$$

This is the nontangential or Stolz approach region. Then Fatou showed that, for $f \in H^{\infty}(D)$ and $\alpha>1$ fixed, the limit

$$
\lim _{\Gamma_{\alpha}(P) \ni z \rightarrow P} f(z)
$$

exists for almost every $P \in \partial D$.

Later on, Privalov [PRI1], [PRI2], Plessner [PLE], and others refined Fatou's result (see $[\mathrm{DIK}]$ for a detailed account of the history). The standard theorem today is that, if $0<p \leq \infty$, if $\alpha>1$ is fixed, and if $f \in H^{p}(D)$ (the Hardy space), then

$$
\lim _{\Gamma_{\alpha}(P) \ni z \rightarrow P} f(z)
$$

exists for almost every $P \in \partial D$. With suitable estimates for the Poisson kernel (see [KRA8]), one can prove a similar result (for nontangential convergence) on any bounded domain in $\mathbb{C}$ with $C^{2}$ boundary. The result may be refined further so that it is valid for functions in the Nevanlinna class (see [GAR] as well as our Section 9).

\footnotetext{
*Received February 8, 2006; accepted for publication September 27, 2006.

$\dagger$ Department of Mathematics, Campus Box 1146, Washington University in St. Louis, St. Louis, MO 63130, USA (sk@math.wustl.edu). It is a pleasure to thank the American Institute of Mathematics for its support and hospitality during a portion of this work.
} 
A theorem of Littlewood (see [LIT]) shows that, in a very precise sense, the nontangential approach regions $\Gamma_{\alpha}$ are the broadest approach regions through which a theorem of this kind can be obtained - even for bounded holomorphic functions.

On a bounded domain in $\mathbb{C}^{n}$ with $C^{2}$ boundary, it is classical that a holomorphic function satisfying suitable growth conditions - say membership in the Hardy class $H^{p}$ or even the Nevanlinna class (see Section 9) - will have nontangential boundary limits almost everywhere with respect to $(2 n-1)$-dimensional area measure $d \sigma$ on the boundary. In the present context, "nontangential" means through an approach region of conical shape:

$$
\Gamma_{\alpha}(P)=\left\{z \in \Omega:|z-P|<\alpha \cdot \delta_{\partial \Omega}(P)\right\},
$$

with $\delta_{\partial \Omega}(P)$ denoting the Euclidean distance of $P$ to $\partial \Omega$. Also, if $\Omega=\left\{z \in \mathbb{C}^{n}\right.$ : $\rho(z)<0\}$, so that $\rho$ is a defining function for $\Omega$ and if $0<p<\infty$, then

$$
H^{p}(\Omega)=\left\{f \text { on } \Omega: \sup _{0<\epsilon<\epsilon_{0}} \int_{\partial \Omega_{\epsilon}}|f(\zeta)|^{p} d \sigma(\zeta)^{1 / p} \equiv\|f\|_{H^{p}(\Omega)}<\infty\right\} .
$$

Also $H^{\infty}(\Omega)$ consists of the bounded holomorphic functions with the supremum norm. Here $\epsilon_{0}$ is a small, positive number and $\Omega_{\epsilon} \equiv\{z \in \Omega: \rho(z)=-\epsilon\}$. Details connected with this definition may be found in [KRA1]. See [ZYG1], [ZYG2], [ZYG3] for historical background of these ideas. The proof of such a theorem depends once again on having the appropriate estimates for the Poisson kernel (see [KRA8], [KRA1]).

It came as quite a surprise when, in 1970, Adam Koranyi [KOR1], [KOR2] showed that a broader method of approach than nontangential is valid when the domain in question is the unit ball in $\mathbb{C}^{n}$. To wit, let $B=\left\{z \in \mathbb{C}^{n}:|z|^{2}<1\right\}$. For $\alpha>1$ and $P \in \partial B$, define

$$
\mathcal{A}_{\alpha}(P)=\{z \in B:|1-z \cdot \bar{P}|<\alpha(1-|z|)\} .
$$

Here, as is standard, $z \cdot \bar{P} \equiv \sum_{j} z_{j} \bar{P}_{j}$. One may calculate (see [KRA1]) that the approach region $\mathcal{A}_{\alpha}$ has nontangential shape in complex normal directions but parabolic shape in complex tangential directions. Koranyi's result is that, if $f \in H^{p}(B)$, then

$$
\lim _{\mathcal{A}_{\alpha}(P) \ni z \rightarrow P} f(z)
$$

exists for $\sigma$-almost every $P \in \partial B$. Koranyi's proof depends decisively on an analysis of the shape of the singularity of the Poisson-Szegö kernel

$$
\mathcal{P}(z, \zeta)=\frac{(n-1) !}{2 \pi^{n}} \frac{\left(1-|z|^{2}\right)^{n}}{|1-z \cdot \bar{\zeta}|^{2 n}} .
$$

for the ball (which shape is decidedly different from the shape of the singularity for the classical Poisson kernel-see [KRA1], [KRA8]). Put in other words, where the classical results depend on estimates for the standard Poisson kernel (in particular, an analysis of its singularity), the new results of Koranyi required estimates on the Poisson-Szegö kernel.

In 1972, E. M. Stein [STE1] showed how to prove a result like Koranyi's on any domain in $\mathbb{C}^{n}$ with $C^{2}$ boundary. His analysis (later refined by Barker-see [BAR] and the discussion in [KRA1]) avoids the use of canonical kernels, but instead depends on an analysis of the Levi geometry of the domain. See also [LEM] for a quite different and original approach to these matters. 
We now realize that Stein's result was an important first step, but it is far from the optimal result for most domains. More precisely, the parabolic approach in complex tangential directions is really only suitable at strongly pseudoconvex boundary points. For points of finite type $m$, an approach region which has aperture $\operatorname{Im} z_{1}=\left|z^{\prime}\right|^{m}$ (where $z_{1}$ is the complex normal direction and $z^{\prime}$ the remaining complex tangential directions - see the discussion below) is the right idea. ${ }^{1}$

But in fact the analysis is much more subtle than that, for it is not just the type of the boundary point but the magnitude of that type that must play a role. The type and the magnitude of the type depend semicontinuously on $P \in \partial \Omega$ when $\Omega \subseteq \mathbb{C}^{2}$. The dependence is more subtle for $\Omega \subseteq \mathbb{C}^{n}, n>2$. The calculations in [NSW1], [NSW2] (see also [KRA9]) begin to show how to tame these rather complicated ideas.

Now let us examine a slightly different direction of these studies. It is an old result of A. P. Calderón [CAL] that if a function $u$, harmonic on the upper halfspace $\mathbb{R}_{+}^{N+1}$, is nontangentially bounded on a set $E \subseteq \mathbb{R}^{N} \equiv \partial \mathbb{R}_{+}^{N+1}$ of positive $N$-dimensional measure then $u$ has nontangential boundary limits at almost every point of E. In the important book [STE1], E. M. Stein proved an analogous result for a holomorphic function on a strongly pseudoconvex domain $\Omega \subseteq \mathbb{C}^{n}$ and for admissible boundedness (see [KRA1] as well as the forthcoming [DIK] for a discussion of both nontangential and admissible approach regions). Of course it is true that a holomorphic function on $\Omega \subseteq \mathbb{C}^{n}$ that is nontangentially bounded on a set $E \subseteq$ $\partial \Omega$ of positive $(2 n-1)$-dimensional measure will have nontangential limits almost everywhere on $E$ - see [CAL], [STE1] and references therein. So certainly a function that is admissibly bounded on a set $E \subseteq \partial \Omega$ will have nontangential limits almost everywhere in $E$, just because admissible boundedness is a stronger condition than nontangential boundedness. One of the main points of the present paper is to give a new proof of a fairly general version of the Calderón theorem for admissible approach regions - one in which the admissible regions have a geometry that is adapted to the particular domain under study (see Section 8). Thus the result presented here is more general than that in [STE1] or [BAR] - just because the approach regions now fit the Levi geometry.

It may be worth noting that a perhaps more general context for some of the results presented here is a space of the form $X \times[0,1]$, where $X$ is a space of homogeneous type (see [COW1], [COW2]). Some relevant references would be [AHN], [[BEL1], [BEL2], [HOR], and [KRL1]. We defer the exploration of this more general situation to another time.

One of the main points of the present work is to make a comparison between nontangential behavior of holomorphic functions of several variables and admissible behavior. We prove the somewhat surprising result that if a holomorphic function on the ball $B \subseteq \mathbb{C}^{n}$ is nontangentially bounded almost everywhere on a set $E \subseteq \partial B$ of positive measure then it is in fact admissibly bounded almost everywhere on $E$. Discussion, context, and proof appear below.

1. Nontangential boundary behavior versus admissible boundary behavior. There are a variety of results in the subject that link, or at least compare and contrast, the isotropic behavior suggested by nontangential approach regions with

\footnotetext{
${ }^{1}$ It is instructive to examine the boundary behavior of a holomorphic function a point $P \in \partial \Omega$ which is strongly pseudoconcave. By the Kontinuitätssatz of multivariable complex function theory (or the Hartogs extension phenomenon), any holomorphic function on $\Omega$ will continue analytically to an entire neighborhood of $P \in \partial \Omega$. So the correct approach region at such a boundary point will be unrestricted. This observation is quite different from the result of Koranyi/Stein.
} 
the nonisotropic behavior suggested by admissible approach regions. Perhaps the first result of this kind was announced by Stein in [STE2]. The details of the argument appear in [KRA1]. The result states that a holomorphic function on a smoothly bounded domain $\Omega$ in $\mathbb{C}^{n}$ which is in a classical Lipschitz function space is in fact in a stronger nonisotropic function space. Roughly speaking, such a function is automatically twice as smooth in tangential directions. This result is in fact valid for all $0<\alpha<\infty$. We now provide some of the concepts and details pertaining to Stein's result.

For $0<\alpha<1$ and $\Omega \subseteq \mathbb{R}^{N}$, we define

$$
\Lambda_{\alpha}(\Omega)=\left\{f: \Omega \rightarrow \mathbb{C}:|f(x+h)-f(x)| \leq C|h|^{\alpha} \text { for all } x, x+h \in \Omega\right\} .
$$

We equip $\Lambda_{\alpha}$ with the norm

$$
\|f\|_{\Lambda_{\alpha}}=\sup _{\substack{x, x+h \in \Omega \\ h \neq 0}} \frac{|f(x+h)-f(x)|}{|h|^{\alpha}}+\|f\|_{L^{\infty}(\Omega)} .
$$

If $\alpha=1$, then we use the slightly more subtle definition

$$
\begin{gathered}
\Lambda_{1}(\Omega)=\{f: \Omega \rightarrow \mathbb{C}:|f(x+h)+f(x-h)-2 f(x)| \\
\leq C|h| \text { for all } x, x+h, x-h \in \Omega\} .
\end{gathered}
$$

We equip $\Lambda_{1}$ with the norm

$$
\|f\|_{\Lambda_{1}}=\sup _{\substack{x, x+h \in \Omega \\ x \neq y}} \frac{|f(x+h)+f(x-h)-2 f(x)|}{|h|}+\|f\|_{L^{\infty}(\Omega)} .
$$

Inductively, if $\alpha>1$, we say that $f \in \Lambda_{\alpha}(\Omega)$ if $f$ is continuously differentiable, $f \in \Lambda_{\alpha-1}$, and $\nabla f \in \Lambda_{\alpha-1}$. The norm is

$$
\|f\|_{\Lambda_{\alpha}}=\|f\|_{\Lambda_{\alpha-1}(\Omega)}+\|\nabla f\|_{\Lambda_{\alpha-1}(\Omega)} .
$$

Assume that the domain $\Omega$ has $C^{2}$ boundary. Let $U$ be a tubular neighborhood of $\partial \Omega$ (see [HIR]). Thus each point in $U$ has a unique nearest point in $\partial \Omega$ and there is a well-defined Euclidean orthogonal projection $\pi: U \rightarrow \partial \Omega$. We say that a $C^{\infty}$ curve $\gamma:[0,1] \rightarrow \Omega \cap U$ lies in $\mathcal{C}^{*}(\Omega)$ if (i) $|\dot{\gamma}(t)| \leq 1$ for all $t$ and (ii) $\dot{\gamma}(t)$ lies in the complex tangent space (see [KRA1]) at $\pi(\gamma(t))$ for each $t$. We think of such a $\gamma$ as a "normalized complex tangential curve". Let $0<\alpha<\infty$. Following E. M. Stein [STE2], we set

$$
\Gamma_{\alpha, 2 \alpha}(\Omega)=\left\{f \in \Lambda_{\alpha}(\Omega): f \circ \gamma \in \Lambda_{2 \alpha}([0,1]) \text { for each } \gamma \in \mathcal{C}^{*}(\Omega)\right\} .
$$

Thus a function in $\Gamma_{\alpha, 2 \alpha}(\Omega)$ is smooth of order $\Lambda_{\alpha}$ in all directions, but smooth of order $\Lambda_{2 \alpha}$ in complex tangential directions.

In fact it is convenient to think about an $f \in \Gamma_{\alpha, 2 \alpha}$ as a function that is $\Lambda_{\alpha}$ along complex normal curves and is $\Lambda_{2 \alpha}$ along complex tangential curves. This point of view has been developed, among other places, in [KRA3]-[KRA6]. See also [GRS] and [RUD].

Stein's remarkable theorem about these function spaces is as follows.

Theorem 1. Let $\Omega \subseteq \mathbb{C}^{n}$ be a domain with $C^{2}$ boundary. Let $\alpha>0$. Let $f$ be a holomorphic function on $\Omega$ which lies in $\Lambda_{\alpha}(\Omega)$. Then $f \in \Gamma_{\alpha, 2 \alpha}(\Omega)$. 
This result has been refined and generalized in [KRA3]-[KRA6]. It is perhaps worth noting that this theorem holds on domains with $C^{2}$ boundary even when $\alpha>1$. This is so because the hypothesis of $C^{2}$ boundary is used only to guarantee that the boundary has a tubular neighborhood.

In [KRA2] it was shown that the analogous result for the space $B M O$ of functions of bounded mean oscillation fails. To wit, if $\Omega=B \subseteq \mathbb{C}^{n}$ is the unit ball, then there are two types of balls to consider in the boundary $\partial B$ : If $P \in \partial B$ and $r>0$ then

$$
\beta_{1}(P, r)=\{z \in \partial B:|z-P|<r\}
$$

and

$$
\beta_{2}(P, r)=\{z \in \partial B:|1-z \cdot \bar{P}|<r\} .
$$

Notice that $\beta_{1}$ is the standard, isotropic Euclidean ball whereas $\beta_{2}$ is a nonisotropic ball with extent $r$ in the complex normal direction and extent $\sqrt{r}$ in the complex tangential directions.

We define

$$
B M O_{1}(\partial B)=\left\{g \text { on } \partial B: \sup _{z, r} \frac{1}{\sigma\left(\beta_{1}(z, r)\right)} \int_{\beta_{1}(z, r)}\left|g(\zeta)-g_{\beta_{1}(z, r)}\right| d \sigma(\zeta)<\infty\right\} .
$$

Here $d \sigma$ is $(2 n-1)$-dimensional Hausdorff measure and $g_{S}$ denotes the average of $g$ over the set $S: g_{S}=[1 / V(S)] \cdot \int_{S} g(t) d V(t)$. Likewise

$$
B M O_{2}(\partial B)=\left\{g \text { on } \partial B: \sup _{z, r} \frac{1}{\sigma\left(\beta_{2}(z, r)\right)} \int_{\beta_{2}(z, r)}\left|g(\zeta)-g_{\beta_{2}(z, r)}\right| d \sigma(\zeta)<\infty\right\} .
$$

Say that $g$ on the ball is in $\mathcal{B M O}_{1}(B)$ if $f$ is holomorphic, $f \in H^{2}$, and $f$ has boundary function that is in fact in $B M O_{1}$. Say that $g$ on the ball is in $\mathcal{B M O}_{2}(B)$ if $f$ is holomorphic, $f \in H^{2}$, and $f$ has boundary function that is in fact in $B M O_{2}$. The result of [KRA2] is that there is a holomorphic function on the ball in $\mathbb{C}^{n}$ which is in classical isotropic $\mathcal{B M O}_{1}$ but not in nonisotropic $\mathcal{B M O}_{2}$. That proof used quite a lot of functional analysis, and did not exhibit the counterexample explicitly. A more concrete proof, with an explicit example, was given in [ULR]. The result of [KRA2] was particularly surprising because it showed as a byproduct that $B M O$ is not an interpolation space between $L^{p}$ and $\Lambda_{\alpha}$.

In view of these results, it is natural to wonder whether a holomorphic function on a domain $\Omega$ in $\mathbb{C}^{n}$ that is nontangentially bounded almost everywhere on a set $E \subseteq \partial \Omega$ will in fact be admissibly bounded almost everywhere on $E$. If this were true, then it would follow, at least for a reasonable class of domains $\Omega$, that a function that is nontangentially bounded on a set $E \subseteq \partial \Omega$ of positive measure will in fact have admissible limits almost everywhere on $E$. In view of the Lindelöf principle developed in $[\mathrm{CIK}]$ and $[\mathrm{KRA} 12]$, this is a very natural sort of result. And it turns out to be true. Its proof is one of the main results of the present paper.

2. Definitions and prior results. We take this opportunity to review the standard definitions and concepts pertaining to this subject. The reference [KRA1] is a good source for the details. See also [KRA11].

We begin with harmonic analysis on $\mathbb{R}^{N+1}$, which is the most natural setting for the consideration of nontangential convergence. Define the upper half space

$$
U=\mathbb{R}_{+}^{N+1}=\left\{x=\left(x_{1}, x_{2}, \ldots, x_{N}, x_{N+1}\right): x_{N+1}>0\right\} .
$$


We often shall write an element of $\mathbb{R}_{+}^{N+1}$ as $\left(x^{\prime}, x_{N+1}\right)$, where $x^{\prime} \in \mathbb{R}^{N}$ and $x_{N+1}>0$. Of course the boundary of $\mathbb{R}_{+}^{N+1}$ can be identified with $\mathbb{R}^{N}=\left\{\left(x_{1}, \ldots, x_{N}, 0\right)\right\}$ in a natural way.

If $\alpha>1$ and $P \in \partial \mathbb{R}_{+}^{N+1} \equiv \mathbb{R}^{N}$, then we define the Stolz region or nontangential approach region $\Gamma_{\alpha}(\mathrm{P})$ of aperture $\alpha$ at $P$ to be

$$
\Gamma_{\alpha}(P)=\left\{x=\left(x^{\prime}, x_{N+1}\right) \in \mathbb{R}_{+}^{N+1}:\left|x^{\prime}-P\right|<\alpha x_{N+1}\right\} .
$$

This is a conical-shaped region in the upper half space. Points in this region cannot approach the boundary along a tangential curve.

Let $f$ be a function on $\mathbb{R}_{+}^{N+1}$. We say that $f$ is nontangentially bounded on a set $E \subseteq \partial \mathbb{R}_{+}^{N+1}$ if, for each $P \in \partial \mathbb{R}_{+}^{N+1}$, there is an $\alpha=\alpha(P)>1$ such that $\left.f\right|_{\Gamma_{\alpha}(P)}$ is bounded. The bound, of course, may (and, in general, will) depend on $P$ and on $\alpha$. But observe that, if $E$ has positive $N$-dimensional measure, then we may use elementary measure theory to find a set $E^{\prime} \subseteq E$ of positive measure and a constant $\alpha^{\prime}>1$ and a number $M^{\prime}>0$ so that $|f| \leq M^{\prime}$ on $\Gamma_{\alpha^{\prime}}\left(P^{\prime}\right)$ for each $P^{\prime} \in E^{\prime}$. Thus we may uniformize the estimate in the definition of "nontangentially bounded".

With notation as in the last paragraph, we say that $f$ has nontangential limit on the set $E \subseteq \partial \mathbb{R}_{+}^{N+1}$ if, for each $\alpha>1$, and each point $P \in E$, the limit

$$
\lim _{\Gamma_{\alpha}(P) \ni x \rightarrow P} f(x)
$$

exists. $^{2}$

Calderón's celebrated theorem [CAL] says this:

Theorem 2. Let $u$ be a harmonic function on $\mathbb{R}_{+}^{N+1}$. Let $E \subseteq \partial \mathbb{R}_{+}^{N+1}$ have positive $N$-dimensional measure. If $u$ is nontangentially bounded on $E$, then $u$ has nontangential limits almost everywhere on $E$.

Calderón notes in his paper that his result holds for holomorphic functions of several complex variables; but that more general result is also formulated in terms of classical nontangential convergence. See also [WID]. In fact the concept of admissible convergence would not be invented for another twenty years.

Let $B \subseteq \mathbb{C}^{n}$ be the unit ball. Let $f$ be a complex-valued function on $B$ and let $E \subseteq \partial B$. We say that $f$ is admissibly bounded on $E$ if, for each $P \in E$, there is an $\alpha=\alpha(P)>1$ such that $f$ is bounded on $\mathcal{A}_{\alpha}(P)$. Using elementary measure theory, it may be seen (in analogy with the situation for classical nontangential convergence) that if $E \subseteq \partial \Omega$ has positive $(2 n-1)$-dimensional measure then there is a set $E^{\prime} \subseteq E$ of positive measure and a number $\alpha^{\prime}>1$ and a constant $M^{\prime}>0$ such that $|f|$ is bounded by $M^{\prime}$ on $\mathcal{A}_{\alpha^{\prime}}\left(P^{\prime}\right)$ for each $P^{\prime} \in E^{\prime}$. See [STE1].

With notation as in the last paragraph, we say that $f$ has admissible limit on the set $E \subseteq \partial \Omega$ if, for each $\alpha>1$, and each point $P \in E$, the limit

$$
\lim _{\mathcal{A}_{\alpha}(P) \ni z \rightarrow P} f(z)
$$

exists.

Now Stein's theorem [STE1, Theorem 12] states the following:

\footnotetext{
${ }^{2}$ It is worth noting that the definition of nontangentially bounded imposes on each $P \in E$ a condition involving just one $\alpha$, depending on $P$. But the definition of nontangential limit imposes on each $P \in E$ a condition for all $\alpha$.
} 
TheOREM 3. Let $\Omega \subseteq \mathbb{C}^{n}$ be a strongly pseudoconvex domain with $C^{2}$ boundary (see [KRA1]). Let $E \subseteq \partial \Omega$ be a set of positive $(2 n-1)$-dimensional measure. Let $f$ be a holomorphic function on $\Omega$. Then $f$ is admissibly bounded on almost everywhere on $E$ if and only if $f$ has admissible limits at almost every point of $E$.

The proof of this last result that appears in [STE1] relies on the potential theory and the Levi geometry of the domain in question. In particular, it requires the construction of a special "preferred" Levi metric. It also depends on estimates involving the Lusin area integral; that is to say, the argument is not direct. S. Ross Barker $[\mathrm{BAR}]$ has provided an alternative, more measure-theoretic approach to the matter and thereby proved the result to be true on a broad class of domains (and also avoided the use of the area integral). Barker only enunciates and proves a result to the effect that a holomorphic function that is admissibly bounded almost everywhere (on the entire boundary) has then admissible limits almost everywhere (on the entire boundary). He comments at the end that his result can be localized (in the spirit of Calderón). One of the points of the present paper is to provide a new approach to the Calderón result. We can also prove a sharper version of the theorem, in the sense that we can in many cases adapt the shape of the approach regions to the Levi geometry of the particular domain under study (see Section 8).

3. The main results of the present paper. In this section we collect the statements of the main results of the present paper. We also briefly indicate their context and significance.

Recall once again that, in the pioneering work [STE1], Stein proves theorems about the boundary behavior of holomorphic functions using approach regions of the same parabolic complex tangential geometry, no matter what the particular intrinsic complex geometry of the domain in question. It was only in later work (see [NSW1], [NSW2], [KRA9]) that the mathematical machinery was developed for adapting the shape of the approach region to the Levi geometry of the domain. The work in [DIB1], [DIB2], [DIB3] extends the new ideas further. It should be stressed that the results presented in the present paper build on these ideas. For instance, the paper [BAR] certainly extends Stein's version of the Calderón local Fatou theorem to any smoothly bounded domain in $\mathbb{C}^{n}$; but it still used the old parabolic approach regions of Koranyi and Stein. In the present paper we prove a version of this theorem for several different types of domains; and we use approach regions that are specifically adapted to the geometry of the domain in question (see Sections 6,8 for the details).

Our theorems do not apply to an arbitrary smoothly bounded domain in $\mathbb{C}^{n}$. At this stage in the development of our mathematical machinery they cannot. For all the proofs here require (i) that the boundary of the domain be equipped with a system of balls that, together with standard $(2 n-1)$-dimensional area measure, make the boundary a space of homogeneous type in the sense of [COW1], [COW2] and (ii) the geometric structure of the approach regions $\mathcal{A}_{\alpha}$ must be compatible (in a sense to be described in detail below) with the balls from (i). As of this writing, we know how to carry out such a program on (a) strongly pseudoconvex domains, (b) domains of finite type in $\mathbb{C}^{2}$, and (c) finite type, convex domains in $\mathbb{C}^{n}$. Refer to Section 8 for the relevant geometric ideas.

TheOREM 4. Let $\Omega$ be either a strongly pseudoconvex domain in $\mathbb{C}^{n}$ or a finite type domain in $\mathbb{C}^{2}$ or a convex, finite type domain in $\mathbb{C}^{n}$. Let $E \subseteq \partial \Omega$ be a set of positive measure (either 3-dimensional Hausdorff measure for a domain in $\mathbb{C}^{2}$ or $(2 n-1)$-dimensional Hausdorff measure for a domain in $\left.\mathbb{C}^{n}\right)$. Suppose that $f$ is a 
holomorphic function on $\Omega$. If $f$ is admissibly bounded at almost every point of $E$ then $f$ has admissible limits at almost every point of $E$.

Discussion: In the strongly pseudoconvex case, Stein proves this theorem in [STE1, Theorem 12]. His proof proceeds by way of a Lusin area integral argument. We provide a new, more direct proof and also extend the result to finite type domains in $\mathbb{C}^{2}$ and convex, finite type domains in $\mathbb{C}^{n}$. We avoid the use of a special "preferred" metric and of the Lusin area integral and work more directly with the Levi geometry of the domain. As part of our treatment of Theorem 4, we shall need to give a detailed consideration of approach regions for Fatou theorems on domains of the type under discussion (Section 6). This is a subtle matter, for the shape of the regions varies in a sort of semi-continuous manner with the base boundary point. Further details will also appear in Section 8 below.

THEOREM 5. Let $f$ be a holomorphic function on the unit ball in $\mathbb{C}^{n}, n>1$. Let $E \subseteq \partial B$ be a set of positive $(2 n-1)$-dimensional measure. Then $f$ is nontangentially bounded at almost every point of $E$ if and only if $f$ is admissibly bounded at almost every point of $E$.

Corollary 6. Let $f$ be a holomorphic function on the unit ball in $\mathbb{C}^{n}, n>1$. Let $E \subseteq \partial B$ be a set of positive $(2 n-1)$-dimensional measure. Assume that $f$ is nontangentially bounded at almost every point of $E$. Then $f$ has admissible limits at almost every point of $E$.

Discussion: In fact this result is valid in considerably greater generality. But all the key ideas are already present in the ball case, and matters are clearer when everything may be written explicitly.

It should be stressed that Theorem 5 is not true point-by-point. That is to say, at a particular point of the boundary of $B$ it is not true that nontangential boundedness implies admissible boundedness. This circle of questions is closely related to the Lindelöf principle, for which see [CIK] and [KRA12].

The theorem answers a fairly old question, one that is rather natural in view of the discussion in Section 1. This new result puts the whole idea of admissible convergence into a very natural context.

4. An ontology of maximal functions. In this paper we shall use eleven different maximal functions. For the convenience of the reader, we collect all their definitions here.

We begin by thinking about the most natural and classical setting for maximal functions, which is the Euclidean space $\mathbb{R}^{N}$. Let $f$ be a locally integrable function on $\mathbb{R}^{N}$. For $x \in \mathbb{R}^{N}$ we define

$$
M f(x)=\sup _{r>0} \frac{1}{|B(x, r)|} \int_{B(x, r)}|f(t)| d t,
$$

and

$$
\mathcal{M} f(x)=\limsup _{r \rightarrow 0^{+}} \frac{1}{|B(x, r)|} \int_{B(x, r)}|f(t)| d t .
$$

Here, as usual,

- The set $B(x, r)$ is the standard isotropic Euclidean ball in $\mathbb{R}^{N}$ with center $x$ and radius $r>0$. 
- We let $|B(x, r)|$ denote the $N$-dimensional Lebesgue measure of $B(x, r)$, which is $c_{N} r^{N}$.

- The measure $d t$ is the standard Lebesgue measure.

It is also useful to let

$$
M_{\delta} f(P)=\sup _{0<r \leq \delta} \frac{1}{|B(x, r)|} \int_{B(x, r)}|f(t)| d t .
$$

The first maximal operator $M$ is the classical one due to Hardy and Littlewood. The second $\mathcal{M}$ is our first new maximal operator. This maximal function differs from the classical one in that the supremum has been replaced by the limit supremum. The third maximal operator $M_{\delta}$ is another small modification of $M$, restricting to balls of radius not exceeding $\delta$.

Now let $\Omega \subseteq \mathbb{C}^{n}$ be a domain on which a notion of admissible approach region $\mathcal{A}_{\alpha}(P), P \in \partial \Omega$, has been defined-see Section 8. If $g$ is a complex-valued function on $\Omega$ and $P \in \partial \Omega$ then we define

$$
g_{\alpha}^{*}(P)=\sup _{z \in \mathcal{A}_{\alpha}(P)}|g(z)|
$$

and

$$
g_{\alpha}^{* *}(P)=\limsup _{\mathcal{A}_{\alpha}(P) \ni z \rightarrow P}|g(z)| .
$$

Now let $B \subseteq \mathbb{C}^{n}$ be the unit ball. Of course $\partial B$ is equipped with a family of isotropic Euclidean balls

$$
\beta_{1}(P, r)=\{z \in \partial B:|z-P|<r\} .
$$

We shall also utilize the nonisotropic balls given by the condition

$$
\beta_{2}(P, r)=\{z \in \partial B:|1-z \cdot \bar{P}|<r\} .
$$

Corresponding to these two types of balls in $\partial B$ we shall have two types of maximal functions. Let $d \sigma$ be boundary area measure. If $\varphi$ is a locally integrable function on $\partial B$ and $P \in \partial B$ then we set

$$
M_{1} \varphi(P)=\sup _{r>0} \frac{1}{\sigma\left(\beta_{1}(P, r)\right)} \int_{\beta_{1}(P, r)}|\varphi(\zeta)| d \sigma(\zeta)
$$

and

$$
M_{2} \varphi(P)=\sup _{r>0} \frac{1}{\sigma\left(\beta_{2}(P, r)\right)} \int_{\beta_{2}(P, r)}|\varphi(\zeta)| d \sigma(\zeta) .
$$

We also define two maximal functions based on the limsup rather than the supremum:

$$
\mathcal{M}_{1} \varphi(P)=\limsup _{r \rightarrow 0^{+}} \frac{1}{\left|\beta_{1}(P, r)\right|} \int_{\beta_{1}(P, r)}|\varphi(t)| d \sigma(t)
$$

and

$$
\mathcal{M}_{2} \varphi(P)=\limsup _{r \rightarrow 0^{+}} \frac{1}{\left|\beta_{2}(P, r)\right|} \int_{\beta_{2}(P, r)}|\varphi(t)| d \sigma(t) .
$$


In addition, we shall have two sets of truncated maximal operators as follows:

$$
M_{1, \delta} \varphi(P)=\sup _{0<r \leq \delta} \frac{1}{\sigma\left(\beta_{1}(P, r)\right)} \int_{\beta_{1}(P, r)}|\varphi(\zeta)| d \sigma(\zeta)
$$

and

$$
M_{2, \delta} \varphi(P)=\sup _{0<r \leq \delta} \frac{1}{\sigma\left(\beta_{2}(P, r)\right)} \int_{\beta_{2}(P, r)}|\varphi(\zeta)| d \sigma(\zeta) .
$$

5. Some estimates for maximal functions. In this section we study some of our new maximal functions. These functions are rather natural tools for the study of the boundary behavior of holomorphic functions. Previous studies (see [STE1], [KRA1], [KRA9], [NSW1], [NSW2]) endeavored to study the entire boundary at once - using classical maximal functions that were designed for such a purpose. Our goal here is to localize the process. This change is particularly propitious for the development of results of Calderón type.

Proposition 7. The maximal operator $\mathcal{M}$ is of weak type $(1,1)$ and also of strong type $(p, p)$ for $1<p \leq \infty$.

Proof. The classical Hardy-Littlewood maximal function $M$ is known (see [STE3]) to be of weak type $(1,1)$ and of strong type $(p, p)$ for $1<p \leq \infty$. Clearly $\mathcal{M} f(x) \leq$ $M f(x)$ for any $f$. The result follows.

We have formulated and proved Proposition 7 on $\mathbb{R}^{N}$. But the statement and proof transfer grosso modo to the boundary of a $C^{2}$, bounded domain in $\mathbb{R}^{N}$ or $\mathbb{C}^{N}$. After all, such a boundary is a smooth manifold hence is locally Euclidean. Put in different terms, this boundary is certainly a space of homogeneous type (see [COW1], [COW2]) when it is equipped with isotropic balls and the standard Hausdorff measure on the boundary. Observe that, thus far, we are not taking the complex structure or the Levi geometry into account. We are only looking at classical Euclidean geometry.

Our key tool in proving boundary limit theorems for holomorphic functions is as follows.

THEOREM 8. Let $\Omega$ be a bounded domain in $\mathbb{C}^{n}, n \geq 2$, with $C^{2}$ boundary which is of one of these types:

- Strongly pseudoconvex domains in $\mathbb{C}^{n}$;

- Finite type domains in $\mathbb{C}^{2}$;

- Finite type, convex domains in $\mathbb{C}^{n}$.

Let $u$ be a real-valued, nonnegative, plurisubharmonic function on $\Omega$, continuous on $\bar{\Omega}$. Let $\varphi=\left.u\right|_{\partial \Omega}$ be the boundary trace of $u$. Let $P \in \partial \Omega$ and $\alpha>1$. Then

$$
u_{\alpha}^{* *}(P) \leq C_{\alpha} \cdot \mathcal{M}_{2} \mathcal{M}_{1} \varphi(P) .
$$

This is our local version of Lemma 8.6.10 in [KRA1] or Theorem 2, p. 11, the Lemma, p. 33, and Lemma 1b, p. 42 in [STE1]. It will be the key tool in obtaining a suitable version of Calderón's theorem for domains in $\mathbb{C}^{n}$. Note that the maximal functions on the righthand side of $(\star)$ are the new localized maximal functions defined in terms of the limit supremum. Thus the are smaller than the maximal functions in the classical inequalities of Stein and Barker.

As we know, once a result like Theorem 8 is established, then it is a straightforward exercise with measure theory to see that suitably bounded holomorphic functions 
have boundary limits. What is new here is the local nature of the maximal function estimate. This, coupled with the newly defined maximal functions, will give a new way to think about Fatou-type theorems and Calderón-type theorems even in $\mathbb{C}^{1}$.

We note that Theorem 8 has of course a standard, classical formulation on the unit disc. In that context, we deal of course with nontangential convergence and there is only one limsup-type maximal function $\mathcal{M}$ on the boundary (see [KRA1]). The estimate then reads

$$
u_{\alpha}^{* *}(P) \leq C^{\prime \prime} \mathcal{M} u(P)
$$

Here we interpret $u_{\alpha}^{* *}$ on the left to be the limsup over $\Gamma_{\alpha}(P)$.

6. Proof of Theorem 8 on the disc and the ball. To fix ideas, we will begin by proving Theorem 8 on the $\operatorname{disc} D$ in $\mathbb{C}$. Now of course the correct concept is classical nontangential convergence, and the function $u$ is subharmonic. Fix a point $P \in \partial D$. We may as well suppose that $P=1+i 0$. Fix a parameter $\alpha>1$ and let $z \in \Gamma_{\alpha}(P)$ be near the boundary. Let $\delta=1-|z|$. For a suitable $c>0$, depending on $\alpha$, we may be sure that $D(z, c \delta) \subseteq D$. Thus

$$
u(z) \leq C \cdot M_{\delta} u(\pi(z))
$$

where $\pi(z)=z /|z|$ is the standard Euclidean projection of $z$ to $\partial D$. See [KRA1, Proposition 8.1.10] or [STE1] for the idea behind estimating $u$ by the classical maximal function on the boundary.

It is essential at this point to notice that the arc centered at $\pi(z)$ and having radius $c^{\prime} \delta$ will certainly contain $P$. This is because $z \in \Gamma_{\alpha}(P)$. Note that $c^{\prime}=c^{\prime}(\alpha)$. Hence we may estimate the last line by

$$
u(z) \leq C^{\prime \prime} \cdot M_{c^{\prime} \delta} u(P) .
$$

Since we are now working on the unit disc $D$ in $\mathbb{C}$, we no longer have distinct maximal functions (modeled on the limsup) based on either isotropic balls or nonisotropic balls. There is just the single limsup maximal function $\mathcal{M}$ based on arcs in $\partial D$.

Now choose a sequence $z_{j} \in \Gamma_{\alpha}(P)$ such that

$$
z_{j} \rightarrow P \quad \text { and } \quad u\left(z_{j}\right) \rightarrow \limsup _{\Gamma_{\alpha}(P) \ni z \rightarrow P} u(z) \equiv u_{\alpha}^{* *}(P)
$$

Then we know by $(\star \star)$ that

$$
u\left(z_{j}\right) \leq C^{\prime \prime} \cdot M_{c^{\prime} \delta_{j}} u(P)
$$

Here $\delta_{j}=1-\left|z_{j}\right|$. Certainly, since $z_{j} \rightarrow P$, we know that $\delta_{j} \rightarrow 0$. As $j \rightarrow \infty$, the righthand side is certainly $\leq C^{\prime \prime \prime} \cdot \mathcal{M} u(P)$. We conclude therefore that

$$
u_{\alpha}^{* *}(P) \leq C^{\prime \prime} \mathcal{M} u(P)
$$

That is the desired conclusion.

Now let us turn to the situation on the ball $B \subseteq \mathbb{C}^{n}$. This circumstance is rather more delicate, for we cannot pass directly from the interior to the boundary by way of single maximal function in order to get the estimates that we need. In the end, the estimate that we obtain is in terms of two maximal functions. 
The nonisotropic balls mesh nicely with the admissible approach regions

$$
\mathcal{A}_{\alpha}(P)=\{z \in B:|1-z \cdot \bar{P}|<\alpha(1-|z|)\} .
$$

Notice in particular that the set of points in $\mathcal{A}_{\alpha}(P)$ having distance precisely $\delta>0$ from $\partial B$ can be described by

$$
\mathcal{E}_{\alpha}(\delta)=\left\{z \in B: \delta_{\partial B}(z)=\delta,|1-z \cdot \bar{P}|<\alpha \cdot \delta\right\} .
$$

The projection of $\mathcal{E}_{\alpha}(\delta)$ to $\partial B$ is the set

$$
\{z \in \partial B:|1-z \cdot \bar{P}|<\alpha \cdot \delta\} .
$$

Thus we see in a natural way that the admissible approach region $\mathcal{A}_{\alpha}(P)$ is built up from the nonisotropic balls $\beta(P, r)$ and, conversely, the nonisotropic balls are projections of level sets of the approach regions $\mathcal{A}_{\alpha}(P)$. It is this relationship, between balls and approach regions, that we shall want to exploit when we study more general domains.

Another key ingredient of our analysis on the unit ball in $\mathbb{C}^{n}$ is the existence of certain polydiscs. If $\alpha>1$ is fixed and $P \in \partial B$, then consider a point $z \in \mathcal{A}_{\alpha}(P)$. It is helpful to normalize coordinates so that $\operatorname{Re} z_{1}$ is the real normal direction at $z$ and thus $\operatorname{Im} z_{1}$ is the complex normal direction. Thus $z_{2}, \ldots, z_{n}$ span the complex tangential directions at $z$. If $\alpha^{\prime}=2 \alpha$ then of course $z \in \mathcal{A}_{\alpha^{\prime}}(P)$. Thus, letting $\delta=1-|z|$, we see that the polydisc

$$
\mathcal{D}=\mathcal{D}(z) \equiv D\left(z_{1}, \delta / 2\right) \times D\left(z_{2},, \sqrt{\delta /(2 \alpha)}\right) \times \cdots \times D\left(z_{2}, \sqrt{\delta /(2 \alpha)}\right)
$$

lies in $\mathcal{A}_{\alpha^{\prime}}$ and hence in $B$.

Now, as usual, let $u$ be a nonnegative function that is continuous on $\bar{B}$ and plurisubharmonic on $B$. Certainly we have (iterating the sub-mean value property on each coordinate disc in each dimension that makes up $\mathcal{D}$ )

$$
u(z) \leq \frac{1}{|\mathcal{D}|} \int_{\mathcal{D}} u(\zeta) d V(\zeta) .
$$

Now, in order to pass from the interior to the boundary, we must exploit our knowledge of the classical Poisson integral. Let us denote the Poisson kernel by $P$ and the Poisson integral of a boundary function $f$ by $P f$. It follows from the maximum principle that the plurisubharmonic function $u$ is majorized by the Poisson integral $P \varphi$ of its boundary function $\varphi$. And that in turn is majorized by (see [KRA1, Chapter 8]) the Hardy-Littlewood maximal function $M_{1} \varphi$ of $\varphi$ at the projected boundary point of the argument; but we in fact only need the Hardy-Littlewood maximal function based on balls of radius $\leq \delta$, and that we denote by $M_{1, \delta}$. Thus line $(*)$ is majorized by

$$
\frac{1}{|\mathcal{D}|} \int_{\pi(\mathcal{D})} M_{1, \delta} \varphi(\pi(\zeta)) \cdot \delta d \sigma(\zeta)
$$

Here, of course, $\pi(z)=z /|z|$ is the projection of $B \backslash\{0\}$ to $\partial B$ and the extra $\delta$ in the integrand comes from the real normal dimension of $\mathcal{D}$.

Now it is essential to note that $\pi(\mathcal{D})$ is comparable to a nonisotropic ball of center $\pi(z)$ and radius $c \delta$. So we may rewrite our estimate as

$$
u(z) \leq \frac{C}{\sigma(\beta(\pi(z), c \delta)} \int_{\beta(\pi(z), c \delta)} M_{1, \delta} \varphi(\zeta) d \sigma(\zeta) .
$$


Because the boundary is a space of homogeneous type - in particular the enveloping property is valid - we may replace the ball of radius $c \delta$ and centered at $\pi(z)$ with a ball of radius $c^{\prime} \delta$ and centered at $P$. So we have

$$
u(z) \leq \frac{C^{\prime}}{\sigma\left(\beta\left(P, c^{\prime} \delta\right)\right)} \int_{\beta\left(P, c^{\prime} \delta\right)} M_{1, \delta} \varphi(\zeta) d \sigma(\zeta) .
$$

And this line is not greater than

$$
C^{\prime} M_{2, \delta}\left(M_{1, \delta} \varphi\right)(P),
$$

where $M_{2, \delta}$ is the Hardy-Littlewood-type maximal function modeled on the nonisotropic balls $\beta$ of radius not exceeding $\delta$. Now choose a sequence $z_{j} \in \mathcal{A}_{\alpha}(P)$ such that $u\left(z_{j}\right) \rightarrow \lim \sup _{z \rightarrow P} u(z)$. Then of course

$$
u\left(z_{j}\right) \leq C^{\prime} M_{2, \delta_{j}}\left(M_{1, \delta_{j}} \varphi\right)(P) .
$$

Letting $j \rightarrow \infty$, we find that

$$
u_{\alpha}^{* *}(P) \leq C^{\prime} \cdot \mathcal{M}_{2} \mathcal{M}_{1} \varphi(P) .
$$

Of course the maximal functions $\mathcal{M}_{j}$ on the right denotes the "limsup" maximal function that we defined and considered earlier. Thus we have obtained the desired estimate.

We have only presented the proof of Theorem 8 so far on the unit ball $B$ in $\mathbb{C}^{n}$. But we assert that it is valid on more general classes of domains, as we have indicated above. In Section 8 we isolate those geometric properties that are needed in order to see that the result goes through in the claimed greater generality.

7. The (Localized) Calderón Theorem. Now we shall present our new approach to the Calderón theorem. To repeat, this point of view is new even in the classical setting of the unit disc in $\mathbb{C}$. We shall confine our discussion to the unit ball, where all the key ideas are already clear.

Proposition 9. Let $f$ be a holomorphic function on the unit ball $B \subseteq \mathbb{C}^{n}$. Let $M>0$ and suppose that $|f| \leq M$. Let $E \subseteq \partial B$ be a set of positive measure, and supposed that $f$ is admissibly bounded on $E$. Then $f$ has admissible limits at almost every point of $E$.

REMARK. The tauberian condition $|f| \leq M$ is a bit artificial, and is certainly not part of the standard canon of the Calderón theorem. But it is a useful tool in our proof. Afterward, we shall remove this condition and recover the standard Calderón result.

Proof. Let $\sigma$ be the usual rotationally-invariant $(2 n-1)$-dimensional area measure on $\partial B$. Let $\epsilon>0$. By outer regularity, select an open set $U \subseteq \partial B$ such that $U \supseteq E$ and $\sigma(U \backslash E) \leq \epsilon \cdot \sigma(E)$. We shall use the maximal functions, and the attendant notation, that we introduced earlier in Section 4.

As usual, if $u$ is a plurisubharmonic function on $B$, continuous on $\bar{B}$, and if $\varphi$ is the boundary trace of $u$, then we know for each $P \in \partial B$ that

$$
u_{\alpha}^{* *}(P) \leq C_{\alpha} \mathcal{M}_{2} \mathcal{M}_{1} \varphi(P) .
$$


Fix a point $P \in \partial \Omega$ and let $\nu=\nu_{P}$ be the unit outward normal vector at $P$. Following the classical argument presented in [KRA1, Theorem 8.6.11], we apply this last inequality to the function

$$
f_{j, k}(z)=\left|f\left(z-\frac{1}{j} \nu_{z}\right)-f\left(z-\frac{1}{k} \nu_{z}\right)\right|
$$

for some $j, k$ positive integers. Then of course $f_{j, k}$ is plurisubharmonic. If we restrict attention to $f$ and $f_{j, k}$ on a neighborhood $\widetilde{\Omega} \cap \Omega$, where $\widetilde{\Omega}$ is a neighborhood in $\mathbb{C}^{n}$ of $P$, then we may also take $f_{j, k}$ to be continuous on $\widetilde{\Omega} \cap \Omega$. Following the argument in the proof of Theorem 8.6.10 in [KRA1], we know that

$$
\int_{E}\left|\left(f_{j, k}\right)_{\alpha}^{* *}(\zeta)\right|^{2} d \sigma(\zeta) \leq C_{\alpha} \cdot \int_{U} \mathcal{M}_{2}\left(\mathcal{M}_{1} f_{j, k}(\zeta)\right)^{2} d \sigma(\zeta)
$$

It is important to note that the maximal functions on the right are defined using the limsup. Thus we may say not only that each maximal function is bounded on $L^{2}$, but also that it is bounded from $L^{2}$ of any open set $\widetilde{U}$ containing $\bar{U}$ to $L^{2}$ of $U$-just because the boundedness would be proved using arbitrarily small balls. So we obtain

$$
\int_{E}\left|\left(f_{j, k}\right)_{\alpha}^{* *}(\zeta)\right|^{2} d \sigma(\zeta) \leq C_{\alpha}^{\prime \prime} \int_{\widetilde{U}}\left|f_{j, k}(\zeta)\right|^{2} d \sigma(\zeta)
$$

where $\widetilde{U}$ is an open set in $\partial B$ that contains $\bar{U}$ and such that $\sigma(\widetilde{U} \backslash E)<\epsilon \cdot \sigma(E)$.

Letting $j \rightarrow \infty$ as in (8.6.10.2) of [KRA1], we find that

$$
\int_{E \mathcal{A}_{\alpha}(\zeta) \ni z \rightarrow \zeta} \limsup _{\mid} \mid\left(f(\zeta)-\left.f\left(z-\frac{1}{k} \nu\right)\right|^{2} d \sigma(\zeta) \leq C_{\alpha}^{\prime \prime} \int_{\widetilde{U}}\left|\widetilde{f}(\zeta)-f\left(\zeta-\frac{1}{k} \nu\right)\right|^{2} d \sigma(\zeta)\right.
$$

Here $\widetilde{f}(\zeta)$ denotes the nontangential limit of $f$ at almost every point of $E$, which we know exists a fortiori by Calderón's classical result.

Now of course the trick (on the righthand side) is to write $\widetilde{U}=(\widetilde{U} \backslash E) \cup E$. Thus

$$
R H S=\int_{\widetilde{U} \backslash E}+\int_{E} \equiv I+I I .
$$

The first integral is estimated quite simply by $4 M^{2} \cdot \sigma(\widetilde{U} \backslash E) \leq C \cdot \epsilon \sigma(E)$. Here, of course $C$ depends on $\alpha$ and on $M$. But it does not depend on any of the other parameters that are relevant to our present estimations. In fact if we replace $\epsilon$ by $\epsilon / M^{2}$, then we may remove the dependence on $M$. This will be important later.

So we have

$$
\begin{aligned}
& \int_{E} \limsup _{\mathcal{A}_{\alpha}(\zeta) \ni z \rightarrow \zeta} \mid\left(f(\zeta)-\left.f\left(z-\frac{1}{k} \nu\right)\right|^{2} d \sigma(\zeta)\right. \\
& \leq C_{\alpha}^{\prime \prime} \int_{E}\left|\tilde{f}(\zeta)-f\left(\zeta-\frac{1}{k} \nu\right)\right|^{2} d \sigma(\zeta)+C \epsilon \sigma(E) .
\end{aligned}
$$

And now one can proceed to imitate the argument at the end of the proof of Theorem 8.6.10 in [KRA1] to find that

$$
\sigma\left\{\zeta \in E: \limsup _{\mathcal{A}_{\alpha}(\zeta) \ni z \rightarrow \zeta}|f(z)-\widetilde{f}(\zeta)|>\epsilon\right\} \leq C \cdot \epsilon \cdot \sigma(E) .
$$


We conclude the argument with standard reasoning using elementary measure theory to see that $\lim _{\mathcal{A}_{\alpha}(\zeta) \ni z \rightarrow \zeta} f(z)=\widetilde{f}(\zeta)$.

Our next job is to remove the tauberian hypothesis (i.e., the assumption of a global bound by $M)$.

TheOREm 10. Let $f$ be a holomorphic function on the unit ball $B \subseteq \mathbb{C}^{n}$. Let $E \subseteq \partial B$ be a set of positive measure, and supposed that $f$ is admissibly bounded almost everywhere on $E$. Then $f$ has admissible limits at almost every point of $E$.

Proof. For $\delta>0$ small, let $B_{\delta} \equiv B(0,1-\delta) \subseteq B \subseteq \mathbb{C}^{n}$. For each such $\delta>0$ there is of course a bound $M_{\delta}$ so that $|f| \leq M_{\delta}$ on $\overline{B_{\delta}}$. If $E$ is as in the statement of the theorem, let $E_{\delta}$ be its Euclidean orthogonal projection into $\partial B_{\delta}$. Fix $\epsilon>0$ as before. Choose $\widetilde{U}_{\delta} \supset E_{\delta}$ so that $\sigma\left(\widetilde{U}_{\delta} \backslash E\right)<\left[\epsilon / M_{\delta}^{2}\right] \cdot \sigma(E)$. Then the estimate $(*)$ holds on $B_{\delta}$ with $E_{\delta}$ replacing $E$ (and, implicitly, $\widetilde{U}_{\delta}$ replacing $\widetilde{U}$ ). Now taking the supremum over $\delta>0$, we find that

$$
\begin{aligned}
& \int_{E} \limsup _{\mathcal{A}_{\alpha}(\zeta) \ni z \rightarrow \zeta} \mid\left(f(\zeta)-\left.f\left(z-\frac{1}{k} \nu\right)\right|^{2} d \sigma(\zeta)\right. \\
& \leq C_{\alpha}^{\prime \prime} \int_{E}\left|\widetilde{f}(\zeta)-f\left(\zeta-\frac{1}{k} \nu\right)\right|^{2} d \sigma(\zeta)+C \epsilon \sigma(E) .
\end{aligned}
$$

And now the proof may be completed as in the argument for the last theorem.

A retrospective of the proof just presented shows that we have constructed machinery that allows a standard sort of localization of the classical Fatou theorem. If the ingredients are in place to prove Theorem 8 , then the Calderón theorem follows immediately. Section 8 explains how all these ingredients are present on domains other than the unit ball $B$.

8. Ingredients needed for a proof on a general domain. At this time we do not know how to prove the results considered here on a perfectly arbitrary bounded domain in $\mathbb{C}^{n}$ with $C^{2}$ boundary. In fact our reasoning depends in essential ways (as does the reasoning of Stein and others) on the Levi geometry of the domain. The pertinent desiderata are in fact known to hold on

(i) the unit disc in $\mathbb{C}$;

(ii) the unit ball in $\mathbb{C}^{n}$;

(iii) strongly pseudoconvex domains in $\mathbb{C}^{n}$;

(iv) domains of finite type in $\mathbb{C}^{2}$;

(v) convex domains of finite type in $\mathbb{C}^{n}, n \geq 2$.

We take this opportunity to isolate the essential features of the geometry that are needed for our reasoning, and give references where the reader may verify that these domains do indeed have the required properties. Fix a bounded domain $\Omega \subseteq \mathbb{C}^{n}$ with $C^{2}$ boundary.

(a) The boundary $\partial \Omega$ must be equipped with a family of balls $\beta_{2}(P, r)$. We use the notation $\beta_{1}(P, r)$ to denote the standard, isotropic, Euclidean balls with center $P$ and radius $r$. The ball $\beta_{2}(P, r)$ will typically be nonisotropic and its shape will derive rather naturally from the complex structure and/or the Levi geometry of $\Omega$.

(b) On the boundary of a suitable domain in $\mathbb{C}^{n}$, the balls $\beta_{2}(P, r)$, together with the standard $(2 n-1)$-dimensional Hausdorff area measure $d \sigma$, form a space of homogeneous type in the sense of [COW1], [COW2]. Of course the classical 
Euclidean balls $\beta_{1}(P, r)$ together with $d \sigma$ also form a space of homogeneous type.

(c) The domain $\Omega$ is equipped with a family of approach regions $\mathcal{A}_{\alpha}(P)$ for each $P \in \partial \Omega$ and each $\alpha>1$. Each $\mathcal{A}_{\alpha}(P)$ is an open set in $\Omega$, and $\mathcal{A}_{\alpha}(P) \subseteq$ $\mathcal{A}_{\alpha^{\prime}}(P)$ whenever $\alpha^{\prime}>\alpha$.

(d) The approach regions $\mathcal{A}_{\alpha}(P)$ and the balls $\beta_{2}(P, r)$ are related in the following manner. If $\alpha>1$ is fixed and $\delta>0$ is small then the Euclidean orthogonal projection of

$$
\left\{z \in \mathcal{A}_{\alpha}(P): \delta_{\partial \Omega}(z)=\delta\right\}
$$

to $\partial \Omega$ is comparable to a ball $\beta_{2}(P, c \delta)$. Here, of course, $c$ will depend on $\alpha$. Conversely, the set

$$
\bigcup_{\delta>0}\left\{z \in \Omega: \pi(z) \in \beta_{2}(P, \delta), \delta_{\partial \Omega}(z)=\delta\right\}
$$

is comparable to an approach region $\mathcal{A}_{c \delta}(P)$.

(e) Suppose, after a normalization of coordinates, that $\operatorname{Re} z_{1}$ is the real normal direction at $z, \operatorname{Im} z_{1}$ the complex normal direction, and $z_{2}, \ldots, z_{n}$ form an orthonormal basis for the remaining $(n-1)$ complex tangential directions. There is a $c>0$ with the following property. If $\alpha>1$ is fixed and $z \in \mathcal{A}_{\alpha}(P)$ with $\delta=\delta_{\partial \Omega}(z)$ then there are positive exponents $\lambda_{1}=\lambda_{1}(z), \ldots, \lambda_{n-1}=$ $\lambda_{n-1}(z)$ so that the polydisc

$$
\mathcal{D}(z) \equiv D\left(z_{1}, c \delta\right) \times D\left(z_{2}, c \delta^{\lambda_{1}}\right) \times \cdots \times D\left(z_{n}, c \delta^{\lambda_{n-1}}\right)
$$

still lies in $\Omega$.

(f) A critical property of the polydisc $\mathcal{D}(z)$ in part (e) is that the Euclidean orthogonal projection $\pi(\mathcal{D}(z))$ in $\partial \Omega$ is comparable to a nonisotropic ball $\beta_{2}\left(\pi(z), c^{\prime} \delta\right)$. What is crucial here is that $\delta$ will be the size of this ball in the complex normal direction, and that will automatically determine all the other dimensions of the $(2 n-1)$-dimensional ball.

(g) The ball $\beta_{2}\left(\pi(z), c^{\prime} \delta\right)$ from part (f) is comparable to a ball $\beta_{2}\left(P, c^{\prime \prime} \delta\right)$, where $P$ is as in part (e).

A review of the proofs that we have presented in Sections 5, 6, 7 show that these seven properties are precisely those that we used to establish our results. Thus Theorem 4 is true for the five types of domains described in (i) $-(\mathbf{v})$.

The references for properties (a)-(g) on domains (i)-(iv) are

(i) For the disc, see [KRA1].

(ii) For the ball, see [KRA11], [KRA1], [STE1].

(iii) For strongly pseudoconvex domains in $\mathbb{C}^{n}$, see [KRA1], [STE1], [KRL2].

(iv) For finite type domains in $\mathbb{C}^{2}$, see [NSW1], [NSW2], [NRSW], [CAT].

(v) For convex, finite type domains in $\mathbb{C}^{n}$, see [DIF], [MCN1], [MCN2] and references therein.

9. The Nevanlinna Class. For many purposes, the most natural space of functions on which to consider Fatou-type theorems is the Nevanlinna class. Here, for a fixed bounded domain $\Omega \subseteq \mathbb{C}^{n}$ with $C^{2}$ boundary, we say that $f$ on $\Omega$ lies in $\mathcal{N}^{+}$if (i) $f$ is holomorphic and (ii) $\log ^{+}|f|$ has a harmonic majorant. By a standard lemma that can be found in [STE1] or [KRA1], this definition is equivalent to requiring that

$$
\sup _{0<\epsilon<\epsilon_{0}} \int_{\partial \Omega_{\epsilon}} \log ^{+}|f(\zeta)| d \sigma(\zeta)<\infty .
$$


Here $\Omega_{\epsilon}=\{z \in \Omega: \rho(z)=-\epsilon\}$ for some defining function $\rho$ for $\Omega$ (see [KRA1]) and

$$
\log ^{+} x=\left\{\begin{array}{lll}
0 & \text { if } & x \leq 1 \\
\log x & \text { if } & x>1
\end{array}\right.
$$

Stein's book [STE1] contained rather elaborate and technical arguments to handle the boundary behavior of functions in $\mathcal{N}^{+}$. A few years later, Barker [BAR] provided a much simpler approach. His key ideas was the next lemma. Note also that the case of meromorphic functions in the Nevanlinna class was treated by Neff [NEF1], [NEF2] and Lempert [LEM].

Lemma 11. Let $u$ be a nonnegative, continuous, plurisubharmonic function on $\Omega$ (we do not necessarily mandate that $u$ be continuous on $\bar{\Omega}$ ). Assume that $u$ has a harmonic majorant. [Thus there is a finite, positive measure $\mu$ on $\partial \Omega$ such that

$$
\left.u(z) \leq \int_{\partial \Omega} P(z, \zeta) d \mu(\zeta) \cdot\right]
$$

Here of course $z \in \Omega$ and $P$ is the standard Poisson kernel. Let $\alpha>1$. Then the admissible maximal function

$$
u_{\alpha}^{*}(\zeta) \equiv \sup _{z \in \mathcal{A}_{\alpha}(\zeta)}|u(z)|
$$

for $\zeta \in \partial \Omega$ satisfies

$$
u_{\alpha}^{*}(\zeta) \leq C_{\alpha}\left[M_{2}\left(\left[M_{1}(\mu)\right]^{1 / 2}\right)\right]^{2} .
$$

and hence is finite almost everywhere in $\partial \Omega$.

We note first of all that Barker's lemma is still true if we replace $u_{\alpha}^{*}$ with our maximal function $u_{\alpha}^{* *}$ (defined using the limsup), $M_{1}$ with $\mathcal{M}_{1}$, and $M_{2}$ with $\mathcal{M}_{2}$. Thus we know that

$$
u_{\alpha}^{* *}(\zeta) \leq C_{\alpha}\left[\mathcal{M}_{2}\left(\left[\mathcal{M}_{1}(\mu)\right]^{1 / 2}\right)\right]^{2} .
$$

As Barker notes, in case $f \in \mathcal{N}^{+}$, one may apply this last lemma to the function $u=\log ^{+}|f|$. It follows then that $u_{\alpha}^{* *}$ is finite almost everywhere, and we may then use our standard arguments to see that $f$ has an admissible limit almost everywhere. Thus we have

TheOREM 12. Let $\Omega \subseteq \mathbb{C}^{n}, n \geq 2$, be a bounded domain with $C^{2}$ boundary. Assume that either $\Omega$ is the ball, or a finite type domain in $\mathbb{C}^{2}$, or a convex finite type domain in $\mathbb{C}^{n}$. Suppose that $f \in \mathcal{N}^{+}(\Omega)$. Then $f$ has admissible boundary limits almost everywhere.

10. Nontangential versus admissible approach. Now we shall prove Theorem 5. In fact, following the example that we have already set with our proof of Theorem 4 (see Proposition 9), we shall at first prove a version of the theorem that has an additional tauberian hypothesis.

THEOREM 13. Let $f$ be a holomorphic function on the unit ball $B$ in $\mathbb{C}^{n}, n>1$. Assume that there is a constant $M>0$ so that $|f| \leq M$. Let $E \subseteq \partial B$ be a set of 
positive $(2 n-1)$-dimensional measure. Then $f$ is nontangentially bounded at almost every point of $E$ (with a bound $C$ that is in general, and most interestingly, smaller than $M$ ) if and only if $f$ is admissibly bounded (with the same bound $C$ ) at almost every point of $E$.

As enunciated, we shall work on the domain the ball $B$, and for simplicity and clarity we shall restrict attention to $B \subseteq \mathbb{C}^{2}$. Thus assume that the holomorphic function $f$ on $B$ is nontangentially bounded on the set $E \subseteq \partial B$ of positive 3-dimensional Hausdorff measure. As usual we call the measure $d \sigma$.

With elementary measure-theoretic arguments, we may extract from $E$ a subset of positive measure so that $f$ is nontangentially bounded at each point of the subset with a uniform bound $C$ and on a cone $\Gamma_{\alpha}$ of uniform size -independent of the point. We continue to call this new set $E$. Not that, in general, $C<M-$ that is certainly the most interesting case. We shall show then that $f$ is admissibly bounded with bound $C$.

Now let $P \in \partial B$ be a point of density (with respect to classical, isotropic balls) of $E \subseteq \partial B$, and let $U \subseteq \partial B$ be a small, relatively open neighborhood of $P$. Let us consider a foliation of $U$ by complex tangential curves. Call the curves $\gamma_{w}:(-\epsilon, \epsilon) \rightarrow$ $U$, where $w$ is a 2-dimensional parameter. Let $g_{w}$ denote the image curve of $\gamma_{w}$. Restrict attention now to those $g_{w}$ which intersect $E$ in a set of positive 1-dimensional measure. For each such $g_{w}$, pick a point $\gamma_{w}\left(t_{w}\right)$ that is a point of 1-dimensional density of $g_{w} \cap E$. Let $\epsilon>0$. Choose a neighborhood $I_{w}=\left(t_{w}-\delta_{w}, t_{w}+\eta_{w}\right)$ so that

$$
\frac{\mathcal{H}^{1}\left(\gamma_{w}\left(I_{w}\right) \cap E\right)}{\mathcal{H}^{1}\left(I_{w}\right)}>1-\epsilon .
$$

We may suppose that $t_{w}, \delta_{w}, \eta_{w}$ are rational numbers. Now, with some elementary measure theory, we may focus on a collection of $\gamma_{w}, w$ in a 2-dimensional set of positive measure, so that each of the $I_{w}$ is the same interval $I^{*}$. Give this set of $w$ the name $\mathcal{S}$, and let $s \in \mathcal{S}$ be a 2-dimensional point of density. We fix attention on the point $x_{0}=\gamma_{s}\left(t_{s}\right)$.

We may repeat the preceding arguments using a foliation $\widetilde{\gamma}_{w}$ of $U$ that is still complex tangential but is transverse to $\gamma_{w}$ (remember that we are working in the boundary of the ball $B$ in $\mathbb{C}^{2}$, so the complex tangent space has real dimension 2). This gives rise to a point $\widetilde{s} \in \widetilde{S}$. By elementary measure theory-in particular by Fubini's theorem-we may suppose that $x_{0}=\gamma_{s}\left(t_{s}\right)=\widetilde{\gamma}_{\tilde{s}}\left(\widetilde{t}_{\tilde{s}}\right)=\widetilde{x}_{0}$. We continue to call the point $x_{0}$.

Thus we focus our attention on the curves $\gamma_{w}\left(I^{*}\right)$ for $w \in \mathcal{S}$ and $\widetilde{\gamma}_{\widetilde{w}}\left(\widetilde{I}^{*}\right)$ for $\widetilde{w} \in \widetilde{S}$. We examine an admissible approach region with base point $x_{0}$ as above. Call that region $\mathcal{A}_{\alpha}\left(x_{0}\right)$, some $\alpha>1$. Let $z \in \mathcal{A}_{\alpha}\left(x_{0}\right)$ be near to the boundary-at distance much less than the length of $\widetilde{I}$ or $\widetilde{I}^{*}$. Let $\delta=\delta_{\partial B}(z)$. Now consider, as usual, a nonisotropic polydisc $\mathcal{D}$ centered at $z$, having radius $c^{\prime} \delta$ in the complex normal directions and radii $c^{\prime} \sqrt{\delta}$ in the complex tangential directions, some small $c^{\prime}>0$.

The natural thing to do at this point is to estimate

$$
|f(z)| \leq \frac{1}{|\mathcal{D}|} \int_{\mathcal{D}}|f(\zeta)| d V(\zeta)
$$

Because of our density statements about $\widetilde{I}$ and $\mathcal{S}$, we can estimate this last line by

$$
\left(1-c^{\prime \prime} \epsilon\right) C+c^{\prime \prime} \epsilon \cdot M \text {. }
$$


Since the point $z \in \mathcal{A}_{\alpha}(s)$ was chosen arbitrarily, and since $\epsilon>0$ was arbitrary, we in fact have shown that $f$ is admissibly bounded at $x_{0}$ with bound $C$. Since points of the kind $x_{0}$ are measure-theoretically generic, we now know that we have a set of positive measure in $E$ on which $f$ is admissibly bounded. Again, by elementary measure theory, we may then conclude that $f$ is admissibly bounded at almost all points of $E$. That completes the proof.

It remains to show that our result holds without the tauberian hypothesis $|f| \leq$ $M$. So now let $f$ be nontangentially bounded on a set $E \subseteq \partial B$ of positive measure. As usual, we may take the nontangential approach regions $\Gamma_{\alpha}(P)$ to be of uniform aperture, and the bound $C$ to be uniform.

For $\tau>0$ small, let $B_{\tau}=B(0,1-\tau)$. Then of course $f$ is bounded by some $M_{\tau}$ on $B_{\tau}$. Let $E_{\tau}$ be the projection of $E$ to $\partial B_{\tau}$. Then of course $f$ is nontangentially bounded on $E_{\tau}$ by $C$ (because each approach region $\mathcal{A}_{\alpha}^{\tau}\left(P_{\tau}\right) \subseteq B_{\tau}$ for $P_{\tau} \in E_{\tau}$ is a subset of $\mathcal{A}_{\alpha}(P)$, where $P=\pi\left(P_{\tau}\right)$ ). Since the tauberian hypothesis is in place on $B_{\tau}$, we may conclude that $f$ is admissibly bounded by $C$ on $E_{\tau}$. But now, for each $P \in E$, note that

$$
\mathcal{A}_{\alpha}(P)=\bigcup_{\tau>0 \text { small }} \mathcal{A}_{\alpha}^{\tau}\left(P_{\tau}\right)
$$

where $\mathcal{A}_{\alpha}^{\tau}\left(T_{\tau}\right)$ is the admissible region in $B_{\tau}$ based at the point $P_{\tau}$ (the projection of $P$ to $\partial B_{\tau}$ ). Since $f$ is admissibly bounded by $C$ on each of the approach regions on the right, it follows that $f$ is bounded by $C$ on $\mathcal{A}_{\alpha}(P)$. This reasoning is valid at almost every point $P$ of $E$. The proof is therefore complete.

11. Concluding remarks. The results in this paper are formulated and proved on the ball, on strongly pseudoconvex domains, on finite type domains in $\mathbb{C}^{2}$, and on convex, finite type domains in $\mathbb{C}^{n}$. Other types of domains can be handled with ad hoc arguments. Among those are the bidisc and complete Reinhardt domains like

$$
\Omega_{2, \infty}=\left\{z \in \mathbb{C}^{2}:\left|z_{1}\right|^{2}+2 e^{-1 /\left|z_{2}\right|^{2}}<1\right\} .
$$

A complete theory of Fatou theorems and Calderón theorems, which can treat any bounded $C^{2}$ domain and which fully accounts for its attendant Levi geometry, has yet to be produced. The paper [KRA9] offers a conceptual framework for handling all domains - using the Kobayashi metric as a stepping stone and structural tool-but in practice it is rather difficult to verify all the hypotheses of the results in [KRA9].

We are of the opinion, however, that invariant metrics are the right argot for formulating function theoretic problems and results on arbitrary domains. Such metrics can read the Levi geometry, and they also take into account the way that holomorphic functions in the interior depend on the shape of the domain. We look forward to future work in this direction.

\section{REFERENCES}

[AHN] P. Ahern ANd A. NAGel, Strong $L^{p}$ estimates for maximal functions with respect to singular measures, with applications to exceptional sets, Duke Math. Jour., 53 (1986), pp. 359-393.

[BAR] S. R. BARKER, Two theorems on boundary values of analytic functions, Proc. Am. Math. Soc., 68 (1978), pp. 54-58.

[BEL1] F. Beatrous and S.-Y. Li, A Plessner decomposition along transverse curves, Can. Jour. Math., 5 (1988), pp. 1243-1255. 
[BEL2] F. BEATrous AND S.-Y. LI, On the boundedness and compactness of operators of Hankel type, J. Functional Analysis, 111 (1993), pp. 350-379.

[CAL] A. P. CALDERón, On the behavior of harmonic functions near the boundary, Trans. Am. Math. Soc., 68 (1950), pp. 47-54.

[CAT] D. CATlin, Estimates of invariant metrics on pseudoconvex domains of dimension two, Math. Zeit., 200 (1989), pp. 429-466.

[CIK] J. A. Cima AND S. G. KRANTZ, A Lindelöf principle and normal functions in several complex variables, Duke Math. Jour., 50 (1983), pp. 303-328.

[COW1] R. R. CoIfman AND G. Weiss, Analyse Harmonique Non-Commutative sur Certain Espaces Homogenes, Lecture Notes in Math. 242, Springer-Verlag, Berlin, 1971.

[COW2] R. R. CoIfMAN AND G. Weiss, Extensions of Hardy spaces and their use in analysis, Bull. AMS, 83 (1977), pp. 569-645.

[DIB1] F. Di BIASE, Approach regions and maximal functions in theorems of Fatou type, thesis, Washington University in St. Louis, 1995.

[DIB2] F. Di BiAse, Exotic Convergence in Theorems of Fatou Type, in "Harmonic Functions on Trees and Buildings", Adam Koranyi, ed., Contemporary Mathematics, vol. 206, American Mathematical Society, 1997.

[DIB3] F. Di BiASE, Fatou type theorems: Maximal Functions and Approach Regions, Birkhäuser Publishing, Boston, 1998.

[DIF] F. Di BIASE AND B. Fischer, Boundary behaviour of $H^{p}$ functions on convex domains of finite type in $\mathbb{C}^{n}$, Pacific J. Math., 183 (1998), pp. 25-38.

[DIK] F. Di Biase AND S. G. KRAntz, The Boundary Behavior of Holomorphic Functions, Birkhäuser Publishing, Boston, 2006, to appear.

[FAT] P. FAtou, Séries trigonométriques et séries de Taylor, Acta Math., 30 (1906), pp. 335400.

[GAR] J. B. Garnett, Bounded Holomorphic Functions, Academic Press, New York, 1981.

[GRS] P. Greiner and E. M. Stein, Estimates for the $\bar{\partial}$-Problem, Princeton University Press, Princeton, NJ, 1977.

[HIR] M. Hirsch, Differential Topology, Springer-Verlag, New York, 1976.

[HOR] L. HÖRMANDER, $L^{p}$ estimates for plurisubharmonic functions, Math. Scand., 20 (1967), pp. $65-78$.

[HUW] R. Hunt And R. WheEden, On the boundary values of harmonic functions, Trans. Amer. Math. Soc., 132 (1968), pp. 307-322.

[JEK] D. JERISON AND C. KENIG, Boundary behaviour of harmonic functions in nontangentially accessible domains, Adv. Math., 46 (1982), pp. 80-147.

[KOR1] A. KoRAnYI, Harmonic functions on Hermitian hyperbolic space, Trans. A. M. S., 135 (1969), pp. 507-516.

[KOR2] A. KorANYI, Boundary behavior of Poisson integrals on symmetric spaces, Trans. A.M.S., 140 (1969), pp. 393-409.

[KRA1] S. G. Krantz, Function Theory of Several Complex Variables, $2^{\text {nd }}$ ed., American Mathematical Society, Providence, RI, 2001.

[KRA2] S. G. KRANTZ, Holomorphic functions of bounded mean oscillation and mapping properties of the Szegö projection, Duke Math. J., 47 (1980), pp. 743-761.

[KRA3] S. G. KRANTZ, Intrinsic Lipschitz classes on manifolds with applications to complex function theory and estimates for the $\bar{\partial}$ and $\bar{\partial}_{b}$ equations, Manuscripta Math., 24 (1978), pp. 351-378.

[KRA4] S. G. Krantz, Smoothness of harmonic and holomorphic functions, Proc. Symp. Pure Math., 35 (1979), (S. Wainger and G. Weiss, eds.), pp. 63-67.

[KRA5] S. G. Krantz, Characterizations of various domains of holomorphy via $\bar{\partial}-$ estimates and applications to a problem of Kohn, Illinois J. Math., 23 (1979), pp. 267-285.

[KRA6] S. G. Krantz, Lipschitz spaces on stratified groups, Trans. Am. Math. Soc., 269 (1982), pp. 39-66.

[KRA7] S. G. Krantz, Finite type conditions and elliptic boundary value problems, Jour. Diff. Eq., 34 (1979), pp. 239-260.

[KRA8] S. G. Krantz, Estimation of the Poisson kernel, Journal of Math. Analysis and Applications, 302 (2005), pp. 143-148.

[KRA9] S. G. KRANTZ, Invariant metrics and the boundary behavior of holomorphic functions on domains in $\mathbb{C}^{n}$, Jour. Geometric Anal., 1 (1991), pp. 71-98.

[KRA10] S. G. KRANTZ, Fatou theorems old and new: an overview of the boundary behavior of holomorphic functions, Proceedings of an International Conference on Complex Variables held in Seoul, Korea, Journal of the Korean Math. Society, 37 (2000), pp. 139-175. 
[KRA11] S. G. Krantz, Partial Differential Equations and Complex Analysis, CRC Press, Boca Raton, FL, 1992.

[KRA12] S. G. KRANTZ, The Lindelöf principle in several complex variables, preprint.

[KRL1] S. G. KRAnTz And S.-Y. LI, A note on Hardy spaces and functions of bounded mean oscillation on domains in $\mathbb{C}^{n}$, Michigan J. Mathematics, 41 (1994), pp. 51-72.

[KRL2] S. G. KRANTZ And S.-Y. Li, Area integral characterizations of Hardy spaces on domains in $\mathbb{C}^{n}$, Complex Variables, 32 (1997), pp. 373-399.

[LEM] L. LEMPERT, Boundary behavior of meromorphic functions of several complex variables, Acta Math., 144 (1980), pp. 1-26.

[MCN1] J. MCNeal, Convex domains of finite type, J. Funct. Anal., 108 (1992), pp. 361-373,

[MCN2] J. MCNeal, Estimates on the Bergman kernels of convex domains, Advances in Math., 109 (1994), pp. 108-139.

[NRSW] A. NAgel, J.-P. Rosay, E. M. Stein, And S. Wainger, Estimates for the Bergman and Szegö kernels in $\mathbb{C}^{2}$, Ann. of Math., 129 (1989), pp. 113-149.

[NSW1] A. NAGel, E. M. Stein, And S. WAinger, Boundary behavior of functions holomorphic in domains of finite type, Proc. Nat. Acad. Sci. USA, 78 (1981), pp. 6596-6599.

[NSW2] A. NAGEL, E. M. Stein, AND S. WAINGER, Balls and metrics defined by vector fields I: Basic properties, Acta Math., 155 (1985), pp. 103-147.

[NEF1] C. A. NefF, Maximal Function Estimates for Meromorphic Nevanlinna Functions, thesis, Princeton University, 1986.

[NEF2] C. A. NefF, Boundary Convergence of Functions in the Nevanlinna Class, Colloq. Math., 60 (1990), pp. 477-506.

[PLE] A. Plessner, Über die Verhalten analytischer Funktionen am Rande ihres Definitionsbereiches, J. F. M., 159 (1927), pp. 219-227.

[PRI1] I. I. Privalov, Integrale de Cauchy, Saratov, 1919.

[PRI2] I. I. PRIVAlov, Randeigenschaften analytischer funktionen, $2^{\text {nd }}$ ed., VEB Deutscher Verlag der Wissenschaften, Berlin, 1956.

[RUD] W. Rudin, Holomorphic Lipschitz functions in balls, Comment. Math. Helvet., 53 (1978), pp. $143-147$.

[STE1] E. M. Stein, Boundary Behavior of Holomorphic Functions of Several Complex Variables, Princeton University Press, Princeton, 1972.

[STE2] E. M. Stein, Singular integrals and estimates for the Cauchy-Riemann equations, Bull. A.M.S., 79 (1973), pp. 440-445.

[STE3] E. M. SteIn, Singular Integrals and Differentiability Properties of Functions, Princeton University Press, Princeton, NJ, 1970.

[UlR] D. Ullrich, TAuberian theorems For PluRiharmonic FUnCtions which are BMO OR BlOCH, Mich. Math. Jour., 33 (1986), pp. 325-333.

[WID] K. O. WIDMAN, On the boundary behavior of solutions to a class of elliptic partial differential equations, Ark. Mat., 6 (1966), pp. 485-533.

[ZYG1] A. ZYGmund, On the boundary values of functions of several variables. I, Fund. Math., 36 (1949), pp. 207-235.

[ZYG2] A. Zygmund, A remark on functions of several variables, Acta Sci. Math. Szeged, Pars B, 12 (1950), pp. 66-68.

[ZYG3] A. ZYGmund, Note on the boundary values of functions of several variables, Ann. of Math. Studies, 25, Princeton University Press, Princeton, NJ, 1950. 
S. G. KRANTZ 